\title{
Update on the Global Burden of Ischemic and Hemorrhagic Stroke in 1990-2013: The GBD 2013 Study
}

\author{
Valery L. Feigin ${ }^{a}$ Rita V. Krishnamurthi ${ }^{a}$ Priya Parmar ${ }^{a}$ Bo Norrving ${ }^{c}$ \\ George A. Mensah ${ }^{i}$ Derrick A. Bennett ${ }^{d}$ Suzanne Barker-Collo ${ }^{b}$ \\ Andrew E. Moran ${ }^{j}$ Ralph L. Sacco ${ }^{k}$ Thomas Truelsen $^{\mathrm{e}}$ Stephen Davis $^{f}$ \\ Jeyaraj Durai Pandian ${ }^{\text {h }}$ Mohsen Naghavil Mohammad H. Forouzanfar ${ }^{\prime}$ \\ Grant Nguyen $^{m}$ Catherine O. Johnson ${ }^{m}$ Theo Vos ${ }^{\prime}$ Atte Meretoja ${ }^{9}$ \\ Christopher J.L. Murray' Gregory A. Roth ${ }^{n}$ GBD 2013 Writing Group and GBD \\ 2013 Stroke Panel Experts Group

\begin{abstract}
${ }^{a}$ National Institute for Stroke and Applied Neurosciences, Auckland University of Technology and ${ }^{b}$ Clinical Training Programme, School of Psychology, The University of Auckland, Auckland, New Zealand; ' ${ }^{\text {Department }}$ of Clinical Sciences, Neurology, Lund University, Lund, Sweden; ${ }^{d}$ Clinical Trial Service Unit and Epidemiological Studies Unit, Nuffield Department of Population Health, University of Oxford, Oxford, UK; ${ }^{e}$ Department of Neurology, Rigshospitalet, University of Copenhagen, Copenhagen, Denmark; ${ }^{f}$ Professor of Translational Neuroscience,

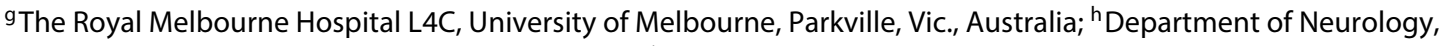
Christian Medical College, Ludhiana, Punjab, India; ${ }^{i}$ Center for Translation Research and Implementation Science and Division of Cardiovascular Sciences; National Heart, Lung, and Blood Institute; National Institutes of Health, Bethesda, Md., jDivision of General Medicine, Columbia University, New York, N.Y., k Department of Neurology, University of Miami Miller School of Medicine, Miami, Fla., 'Department of Global Health, School of Medicine and Public Health, ${ }^{m}$ Institute for Health Metrics and Evaluation, and ${ }^{n}$ Institute for Health Metrics and Evaluation and the Division of Cardiology, School of Medicine, University of Washington, Seattle, Wash., USA
\end{abstract}

\section{Key Words}

Stroke $\cdot$ Ischemic stroke $\cdot$ Hemorrhagic stroke $\cdot$ Global burden. GBD 2013

\section{Abstract \\ Background: Global stroke epidemiology is changing rap- idly. Although age-standardized rates of stroke mortality have decreased worldwide in the past 2 decades, the abso- lute numbers of people who have a stroke every year, and}

live with the consequences of stroke or die from their stroke, are increasing. Regular updates on the current level of stroke burden are important for advancing our knowledge on stroke epidemiology and facilitate organization and planning of evidence-based stroke care. Objectives: This study aims to estimate incidence, prevalence, mortality, disability-

Members of the GBD 2013 Writing Group and GBD 2013 Stroke Panel Experts Group are listed in the Appendix at the end of the paper.

\section{KARGER 125}

(C) 2015 S. Karger AG, Basel

0251-5350/15/0453-0161\$39.50/0

E-Mail karger@karger.com

www.karger.com/ned
Professor Valery L. Feigin, MD, MSc, PhD, FAAN

NISAN, AUT University, AUT North Shore Campus, AA254

90 Akoranga Dr, Northcote 0627

Auckland 1142 (New Zealand)

E-Mail valery.feigin@aut.ac.nz 
adjusted life years (DALYs) and years lived with disability (YLDs) and their trends for ischemic stroke (IS) and hemorrhagic stroke (HS) for 188 countries from 1990 to 2013. Methodology: Stroke incidence, prevalence, mortality, DALYs and YLDs were estimated using all available data on mortality and stroke incidence, prevalence and excess mortality. Statistical models and country-level covariate data were employed, and all rates were age-standardized to a global population. All estimates were produced with $95 \%$ uncertainty intervals (Uls). Results: In 2013, there were globally almost 25.7 million stroke survivors (71\% with IS), 6.5 million deaths from stroke (51\% died from IS), 113 million DALYs due to stroke ( $58 \%$ due to IS) and 10.3 million new strokes (67\% IS). Over the 1990-2013 period, there was a significant increase in the absolute number of DALYs due to IS, and of deaths from IS and HS, survivors and incident events for both IS and HS. The preponderance of the burden of stroke continued to reside in developing countries, comprising $75.2 \%$ of deaths from stroke and $81.0 \%$ of stroke-related DALYs. Globally, the proportional contribution of stroke-related DALYs and deaths due to stroke compared to all diseases increased from 1990 (3.54\% (95\% UI 3.11-4.00) and 9.66\% (95\% UI 8.47-10.70), respectively) to 2013 (4.62\% (95\% UI 4.01-5.30) and $11.75 \%$ (95\% UI 10.45-13.31), respectively), but there was a diverging trend in developed and developing countries with a significant increase in DALYs and deaths in developing countries, and no measurable change in the proportional contribution of DALYs and deaths from stroke in developed countries. Conclusion: Global stroke burden continues to increase globally. More efficient stroke prevention and management strategies are urgently needed to halt and eventually reverse the stroke pandemic, while universal access to organized stroke services should be a priority.

(c) 2015 S. Karger AG, Basel

\section{Introduction}

Stroke remains a major global health problem $[1,2]$ and its significance is likely to increase in the future due to ongoing demographic changes, including aging of the population and health transitions observed in developing countries [3]. The Global Burden of Disease (GBD) 2010 study showed that while age-standardized rates of stroke mortality have decreased worldwide in the past 2 decades, the absolute numbers of people who have a stroke every year, live with the consequences of stroke and die from their stroke, is increasing. New epidemiological papers on stroke published since 2010 and updated death records are now included in the GBD 2013 study data set. Impor- tant advances and refinements have been made to the methodology for deriving the GBD estimates. Therefore, this updated report on the current level of stroke burden is important for advancing our knowledge on stroke epidemiology and planning for adequate evidence-based stroke systems of care. In the GBD 2013 study, we use the new DisMod $[4,5]$ methodology in order to provide new estimates of incidence, prevalence, disability-adjusted life years (DALYs) and years lived with disability (YLDs) for ischemic stroke (IS) and hemorrhagic stroke (HS) and other non-ischemic strokes combined separately for 1990 and 2013 at regional and global levels.

\section{Methodology}

Methods to estimate all-cause mortality and cause-specific mortality have been previously described [6]. Stroke modeling and methodology used to estimate GBD 2013 stroke burden (including an updated list of publications included in this analysis) has also been described in detail [7]. In brief, we used all available estimates of stroke incidence, prevalence and case fatality from systematic reviews of the scientific literature, population surveys and stroke registries. Stroke was defined based on the WHO clinical criteria [8]. All available national mortality data were compiled. Non-specific cause codes were redistributed based on expert opinion and statistical methods [7]. The total for all causespecific deaths was fitted within an envelope for all-cause mortality. Deaths were compiled into 240 causes, including IS and HS. For stroke death estimates, GBD defined stroke ICD-10 codes as IS, HS or non-specific as to type. The parent category of cerebrovascular disease was based on the mapping of the detailed causes. Deaths coded as due to G45 (transient ischemic attack) were coded as IS and deaths coded as due to non-ruptured aneurysms (ICD code I67.0) were coded as HS. Non-specific codes, including I64, I67.9, I68.8, I69.4-I69.9, were redistributed to IS or HS using a regression model.

An ensemble model was used to estimate a continuous time series for mortality by age, sex, country (developed or developing) and year. Country-level covariates associated with stroke were used in the model, and out-of-sample validity testing was used to assess model performance. Also, 95\% uncertainty intervals (UIs) were estimated using 1,000 draws from the posterior distribution for each age-sex-country group. Disease prevalence was estimated using the DisMod state transition disease modeling software [9] and the Bayesian statistical models. IS and HS were modeled separately. Adjustments were made to account for incidence estimates specifying first-ever or any stroke. Disability due to acute stroke was considered to last for 28-30 days and for chronic stroke from 31 st day until death. Five disability weights were derived from large-scale studies while SF-12 scores from the U.S. Medical Expenditures Panel Survey for stroke patients supplied the distribution of these severity levels plus the proportion asymptomatic [10]. YLDs were calculated as the product of a disability weight and prevalent cases of stroke. DALYs were calculated as the sum of years of life lost prematurely, based on maximum observed global longevity and YLDs. Countries were stratified into 2 groups (de- 

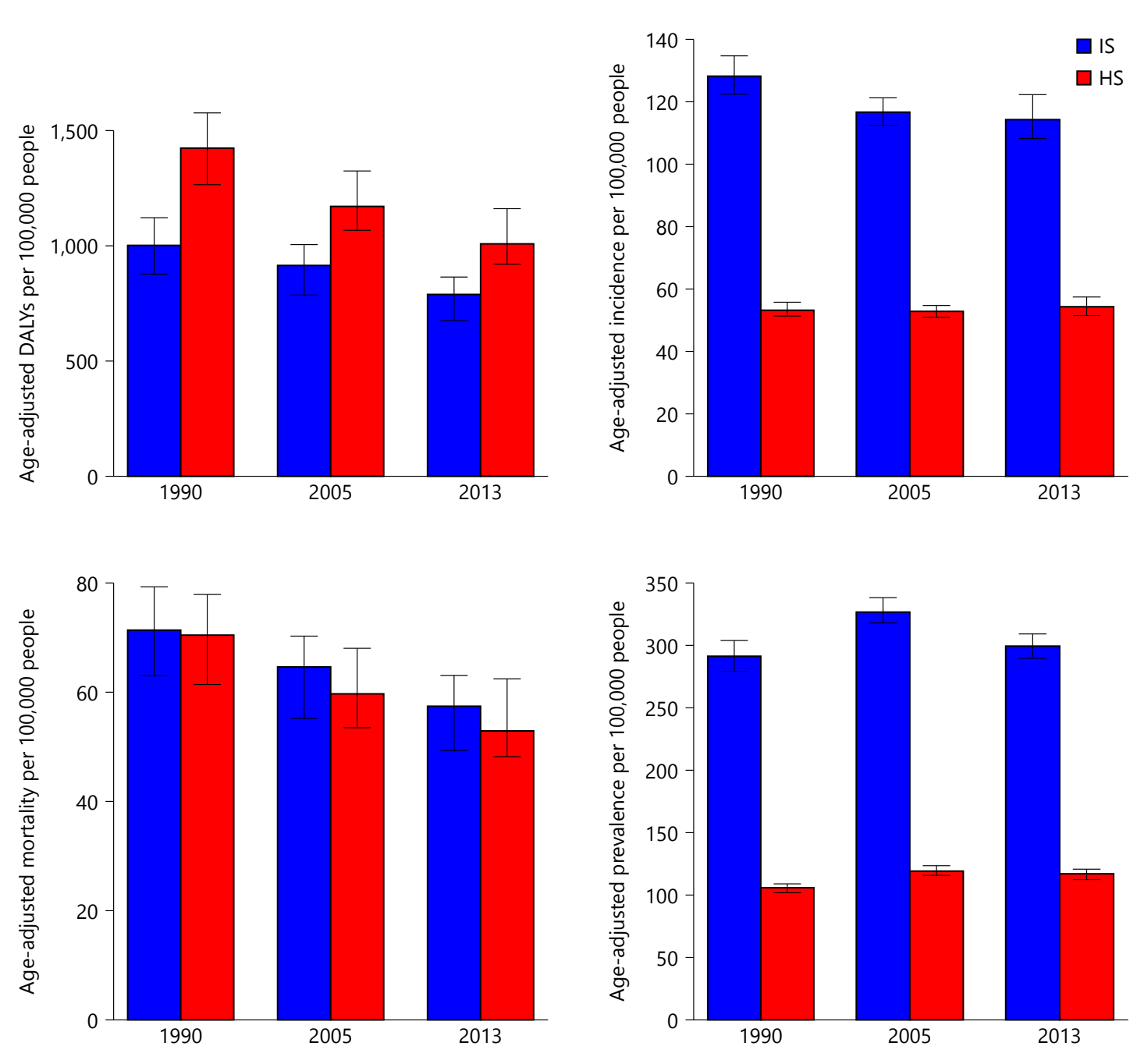

Fig. 1. Age-adjusted DALYs, mortality, incidence and prevalence rates of IS and HS per 100,000 people (with $95 \%$ UIs) in 1990, 2005 and 2013.

veloped countries (high-income countries) and developing countries (low- and middle-income countries)). We report age-standardized incidence and mortality rates per 100,000 person-years, and prevalence and DALY estimates per 100,000 people with the direct method of standardization and GBD estimates of population as a reference.

\section{Results}

Globally from 1990 to 2013, there were statistically significant reductions in the incidence, mortality and DALY rates of IS. For HS, there were statistically non-significant increases in the incidence and prevalence, and decreases in the mortality and DALY rates (fig. 1; table 1). In 2013, the DALYs and mortality rates of IS and HS combined in developing countries $(2,189 / 100,000$ per year $(95 \%$ UI $1,995-2,416 / 100,000$ per year) and $137 / 100,000$ per year (95\% UI 125-150/100,000 per year), respectively) were statistically significantly greater than that in developed countries $(1,022 / 100,000$ (95\% UI $941-1,159 / 100,000)$ and $67 / 100,000$ (95\% UI 62-78/100,000), respectively) due to the higher rate for HS (table 1), while the absolute number of people affected by both IS and HS in the world over that time period had increased significantly (table 2). While mortality rates from IS and HS combined in devel- 
Table 1. Age-adjusted DALYs and mortality rates of IS and HS combined per 100,000 people (with 95\% UIs) in developed and developing countries in 1990 and 2013

\begin{tabular}{llccr}
\hline Year and stroke type & Metric & Developed countries & Developing countries & Globally \\
\hline 1990 & & & & \\
IS and HS combined & DALYs & $1,721.4(1,564.3-1,866.5)$ & $2,818.2(2,578.3-3,064.0)$ & $2,430.8(2,224.0-2,631.4)$ \\
& Mortality & $112.9(100.6-121.8)$ & $160.9(146.8-174.8)$ & $141.6(128.5-153.9)$ \\
IS & DALYs & $1,023.7(906.4-1,120.3)$ & $950.6(807.6-1,096.6)$ & $1,004(877.6-1,222.4)$ \\
& Mortality & $76.8(67.3-84.1)$ & $63.7(54.9-73.8)$ & $71.3(63.0-79.3)$ \\
HS & DALYs & $697.7(598.4-789.4)$ & $1,867.6(1,657.9-2,069.3)$ & $1,426.0(1,269.9-1,579.9)$ \\
& Mortality & $36.1(30.1-41.2)$ & $97.2(84.8-108.6)$ & $70.3(61.2-77.9)$ \\
& Mortality & $27.9(25.1-33.2)$ & $80.9(72.5-93.0)$ & $59.5(53.5-68.1)$ \\
IS and HS combined & & & \\
& DALYs & $1,022.2(940.9-1,158.6)$ & $2,189.3(1,995.4-2,416.0)$ & $1,806.9(1,667.4-1,991.7)$ \\
IS & Mortality & $67.2(61.9-78.2)$ & $136.9(125.2-150.4)$ & $110.1(101.8-122.2)$ \\
& DALYs & $609.8(553.0-707.1)$ & $889.4(718.1-989.8)$ & $791.3(678.0-868.8)$ \\
HS & Mortality & $44.9(40.9-53.5)$ & $65.1(52.1-72.0)$ & $57.3(49.3-62.9)$ \\
& DALYs & $412.4(369.1-473.3)$ & $1,299.9(1,178.2-1,495.9)$ & $1,015.6(923.2-1,163.2)$ \\
& Mortality & $22.2(19.6-26.1)$ & $71.8(64.6-85.6)$ & $52.8(48.0-62.3)$ \\
\hline
\end{tabular}

Table 2. Absolute number of DALYs, deaths, incident and prevalent cases of IS and HS (with 95\% UIs) in the world in 1990 and 2013

\begin{tabular}{llcc}
\hline & Metric & 1990 & 2013 \\
\hline$I S$ & DALYs & $34,155,606(29,592,196-38,325,866)$ & $47,424,681(40,537,540-52,211,800)$ \\
& Deaths & $2,182,865(1,923,290-2,430,872)$ & $3,272,924(2,812,654-3,592,562)$ \\
& Incidence & $4,309,356(4,118,103-4,531,909)$ & $6,892,857(6,549,814-7,352,226)$ \\
& Prevalence & $10,045,202(9,643,525-10,453,439)$ & $18,305,491(17,767,372-18,920,736)$ \\
\hline$H S$ & DALYs & $55,953,376(49,881,127-62,161,971)$ & $65,454,194(59,497,415-74,654,738)$ \\
& Deaths & $2,401,930.40(2,109,380.2-2,669,117.5)$ & $3,173,951(2,885,717-3,719,684)$ \\
& Incidence & $1,886,345(1,816,991-1,976,659)$ & $3,366,175(3,199,978-3,543,213)$ \\
& Prevalence & $3,891,158(3,769,541-4,019,014)$ & $7,363,457(7,139,691-7,616,146)$ \\
\hline
\end{tabular}

oped countries were almost halved from 1990 to 2013 (112.9/100,000 and 67.2/100,000, respectively), IS and HS mortality rates in developing countries were reduced by only approximately 15\% (from 160.9/100,000 in 1990 to $136.9 / 100,000$ in 2013). As shown in table 2, in 2013 there were almost 25.7 million stroke survivors ( $71 \%$ with IS), 6.5 million deaths from stroke (51\% died from IS), 113 million DALYs due to stroke (58\% due to IS) and 10.3 million new strokes (67\% were IS). Over the 1990-2013 period, there was a statistically significant increase in the absolute number of DALYs due to IS, and deaths, survivors and incident events of as a result of both IS and HS. The prevalence nearly doubled for both IS and HS from 1990 to 2013. Globally, from 1990 to 2013, there was a statistically non-significant increase in the prevalence rates of IS (statistically significant increase in the preva- lence of IS from 1990 to 2005 was followed by statistically significant decrease from 2005 to 2013; fig. 1) and a statistically significant increase in HS prevalence rates (fig. 1). However, in developed countries, the increase in the prevalence rates was statistically significant for both IS and HS (table 3).

Analysis of age-specific patterns of incidence, prevalence and mortality rates by country development status in 2013 (fig. 2, 3) showed that IS incidence and prevalence rates in developed countries were statistically significantly greater and demonstrated a steeper increase with age than that in developing countries after the age of 49 and 39 years, respectively. However, IS mortality rates after the age of 49 years were greater in developing countries. The age-related patterns of increase in IS mortality rates were similar in developed and developing countries. Age- 
Table 3. Age-adjusted prevalence rates of IS and HS per 100,000 people and median percentage change with $95 \%$ UIs in developed and developing countries in 1990 and 2013

\begin{tabular}{|c|c|c|c|c|c|}
\hline & Years & \multicolumn{2}{|l|}{ IS } & \multicolumn{2}{|l|}{ HS } \\
\hline Developing countries & $\begin{array}{l}1990 \\
2013\end{array}$ & $\begin{array}{l}155.4(148.6-161.5) \\
156.0(149.3-164.6)\end{array}$ & $\begin{array}{l}0.001(-0.051 \text { to } 0.065) \\
0.214(0.140 \text { to } 0.317)\end{array}$ & $\begin{array}{l}113.8(110.0-117.8) \\
114.3(110.3-118.0)\end{array}$ & $\begin{array}{l}0.003(-0.040 \text { to } 0.049) \\
0.372(0.253 \text { to } 0.521)\end{array}$ \\
\hline Developed countries & $\begin{array}{l}1990 \\
2013\end{array}$ & $\begin{array}{l}472.7(446.0-500.1) \\
577.6(558.7-600.4)\end{array}$ & $0.024(-0.028$ to 0.091$)$ & $\begin{array}{c}93.3(86.4-99.7) \\
128.3(121.1-135.9)\end{array}$ & $0.102(0.054$ to 0.161$)$ \\
\hline Globally & $\begin{array}{l}1990 \\
2013\end{array}$ & $\begin{array}{l}291.2(278.7-303.8) \\
299.1(290.2-309.2)\end{array}$ & & $\begin{array}{l}105.6(102.0-109.2) \\
116.6(113.1-120.5)\end{array}$ & \\
\hline
\end{tabular}

Fig. 2. Age-specific incidence, prevalence and mortality rates of IS per 100,000 people per year by country development status in 2013 (shadowed area around solid lines represents $95 \%$ UIs). IS incidence by age and development status, 2013.

(For figures $b$ and $c$ see next page.)

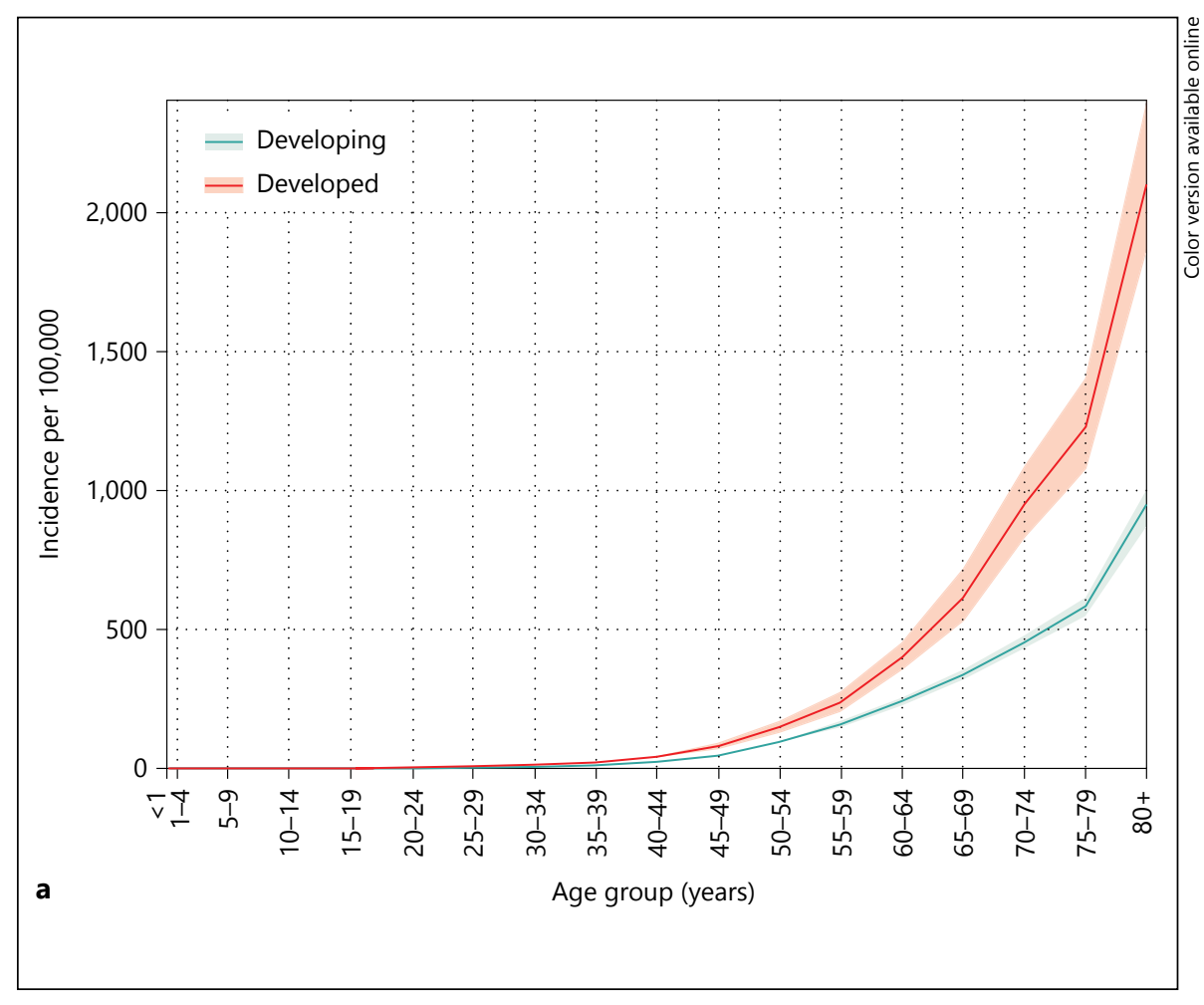

specific incidence and mortality rates of HS were statistically significantly greater in developing countries after the age of 39 years (fig. 3). There was no statistically significant difference in the age-specific prevalence rates between developed and developing countries after 54 years (fig. 3). However, the prevalence rates of HS in younger age groups were significantly greater in developed countries.

Analysis of the percentage contribution of IS and HS to all diseases (table 4) showed that the relative burden of strokes in the world increased from 1990 to 2013, particularly for the burden due to IS in which the increase in DALYs and in deaths reached a statistically significant level. In developing countries, statistically significant increases in DALYs and deaths due to stroke were present for both IS and HS, while there was little change in YLDs. In developed countries, we observed a decrease in the proportional contribution of DALYs and deaths from IS and HS compared to all other diseases but an increase in the proportional contribution of YLDs due to stroke. In 


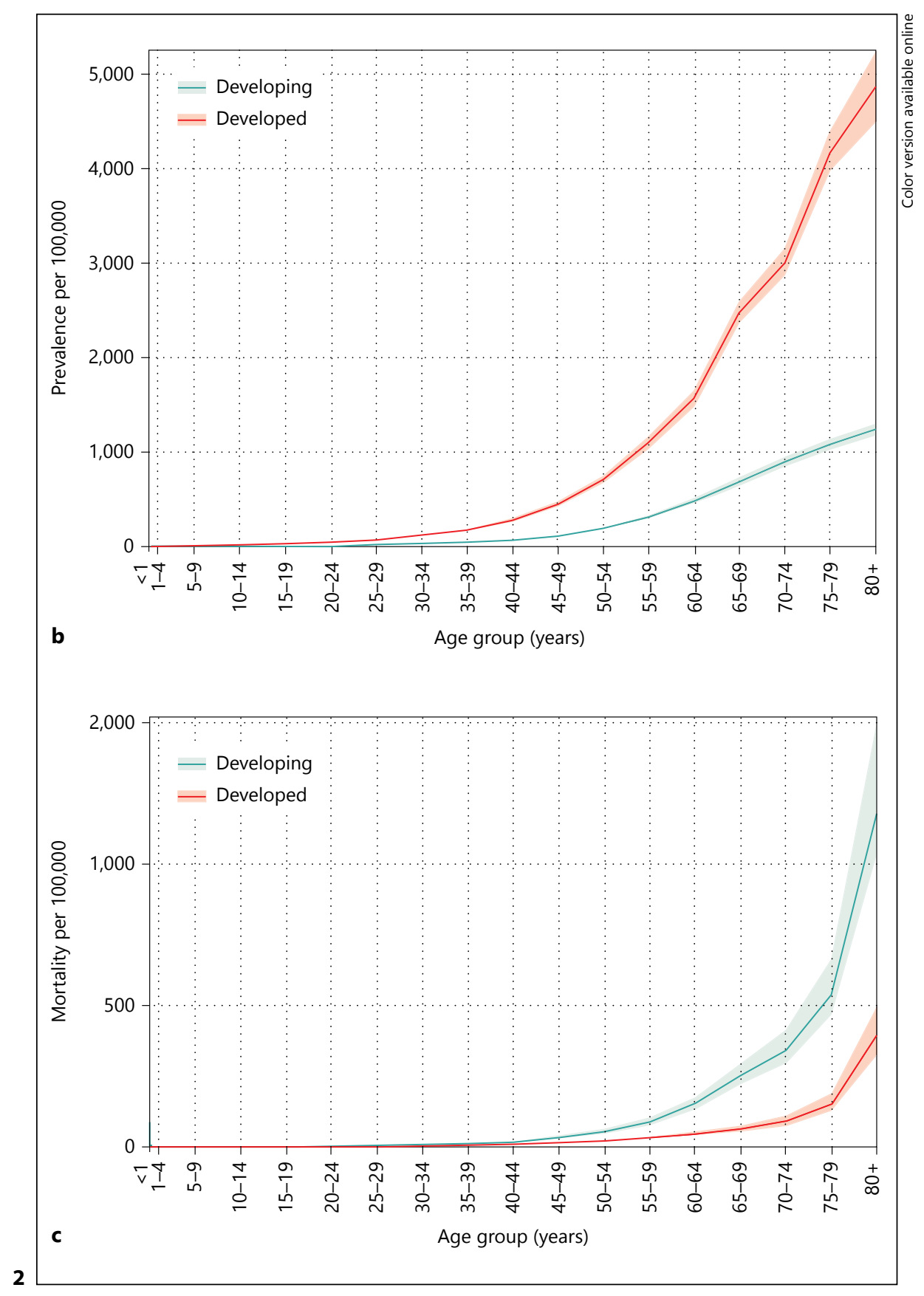

comparison to other causes of DALYs in the world (fig. 4), stroke was the second largest contributor after ischemic heart disease globally and in developing countries, and the third largest contributor to DALYs in developed countries (after ischemic heart disease and lower back and neck pain), with significant regional variation in disease burden across both developed and low- to middleincome countries.
There were large inter- and intra-regional variations in IS and HS age-adjusted incidence, prevalence and mortality rates (fig. 5, 6; online suppl. tables; for all online suppl. material, see www.karger.com/doi/10.1159/ 000441085). The highest prevalence rate of IS $(1,015-$ $1,184 / 100,000)$ was shown in developed countries (particularly in the USA), the lowest (up to $339 / 100,000$ ) in developing countries. The highest IS mortality rates 
Fig. 3. Age-specific incidence, prevalence and mortality rates of HS per 100,000 people per year by country development status in 2013 (shadowed area around solid lines represents 95\% UIs). HS incidence by age and development status, 2013.

(For figure $c$ see next pages.)

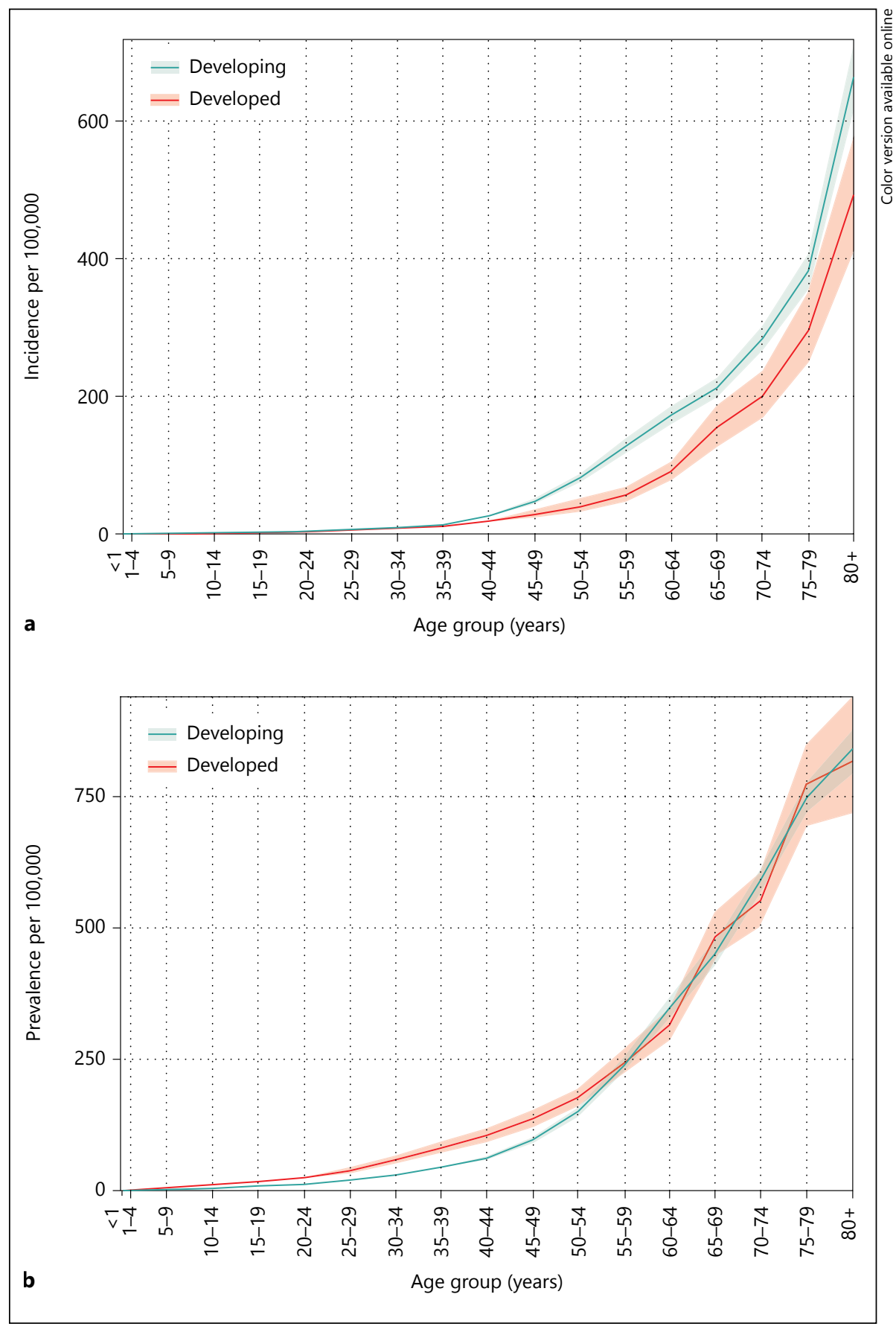

(124-174/100,000 person-years) were observed in Russia and Kazakhstan, and the lowest (at or below 25/100,000) in Western Europe, North and Central America, Turkmenistan and Papua New Guinea. Prevalence rates of HS were highest $(232-270 / 100,000)$ in the USA, and lowest (up to 78/100,000) in Latin America, Africa, Middle East, France, Eastern Europe, Northern part of Asia and Russia. HS mortality rates were highest (159-222/100,000 person-years) in Mongolia and Madagascar, and lowest (up to 32/100,000) in North America, most parts of Western Europe, Russia, Iran, Saudi Arabia, Morocco, Japan, Australia and New Zealand (fig. 6). 


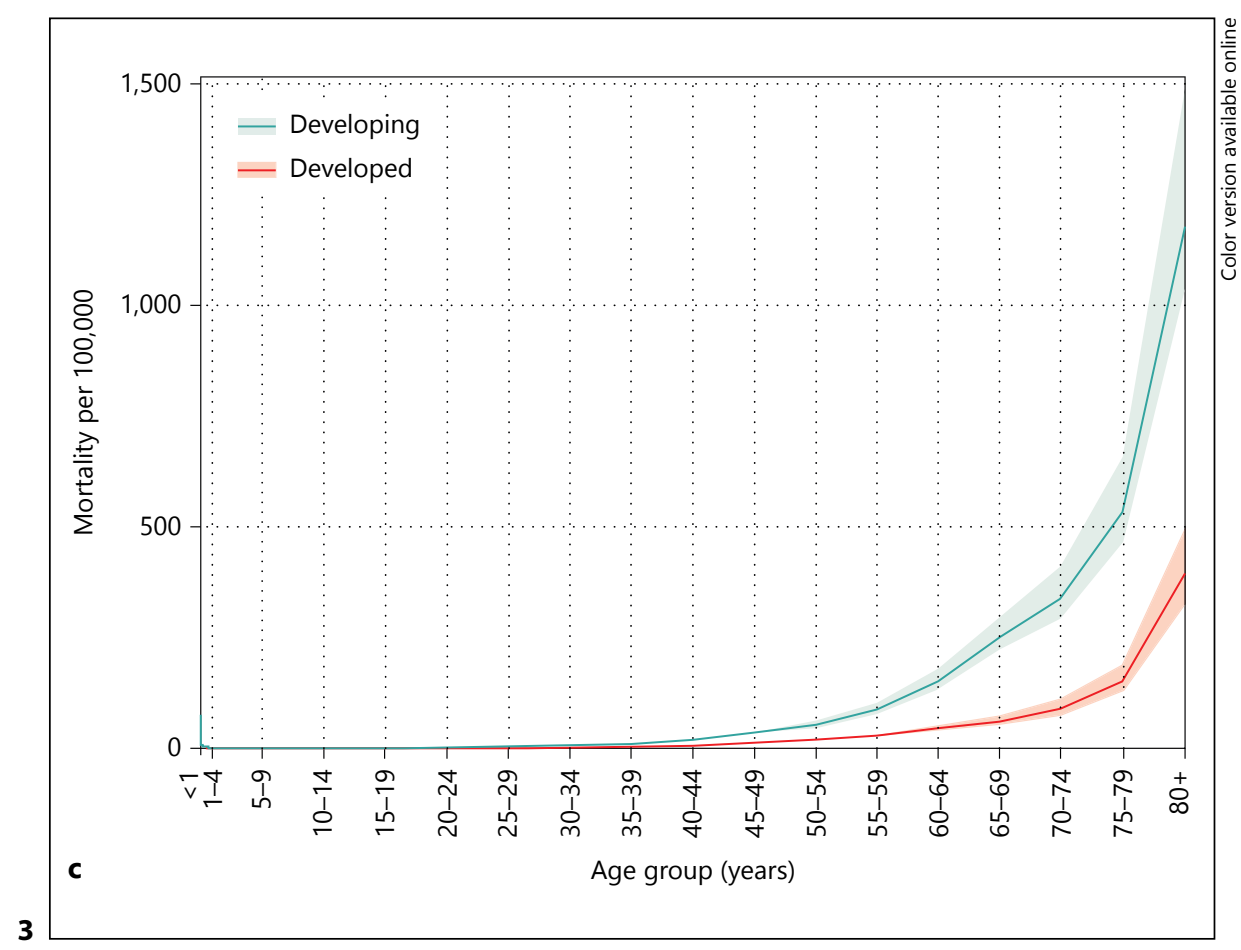

Table 4. Proportional (\%) contribution of IS and HS burden (with 95\% UIs) to all health conditions by year and country development status

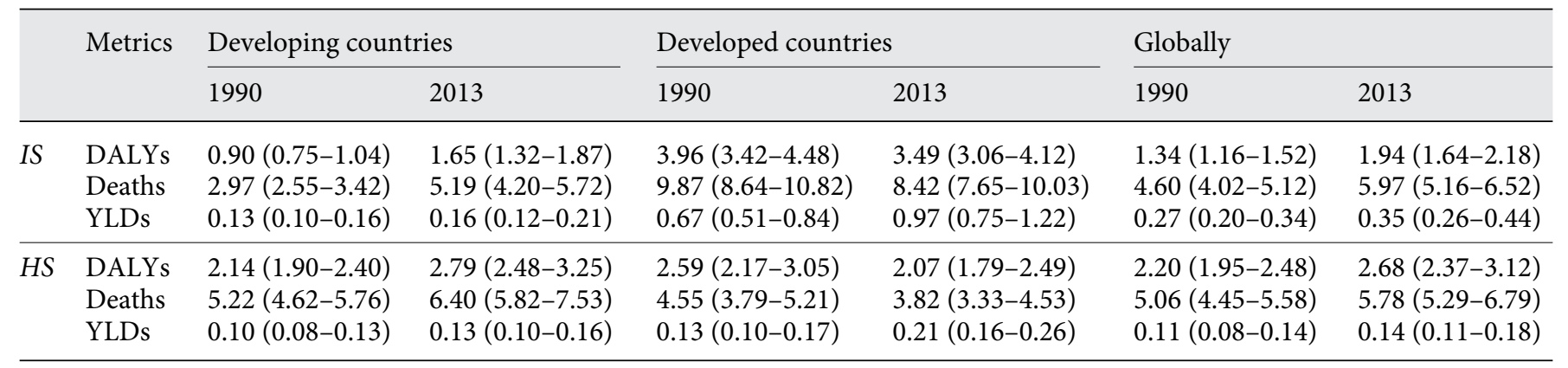

\section{Discussion}

The GBD 2013 stroke study supersedes all previous GBD stroke estimates. These new data confirm the previous GBD observations [1] about the significant increase in stroke burden in the world over the last two decades and substantial geographical differences in stroke burden and the directions of the changes by country income level $[1,11]$. We observed a greater than 3 -fold increase in burden due to stroke with 4.85 million stroke deaths and 91.4 million DALYs in developing countries compared with 1.6 million deaths and 21.5 million DALYs in high- income countries. We demonstrated that in 2013 there were almost 25.7 million stroke survivors ( $71 \%$ with IS), 6.5 million deaths from stroke (51\% died from IS), 113 million DALYs due to stroke (58\% due to IS) and 10.3 million of people with new strokes (67\% were IS). Over the 23-year study period (1990-2013), there was a much greater reduction in stroke mortality rates in developed countries than those in developing countries, thus further deepening the disparities in the global stroke burden.

We showed that the HS incidence and mortality are very high in developing countries. The YLD did not change due to HS mainly because of high mortality rate 


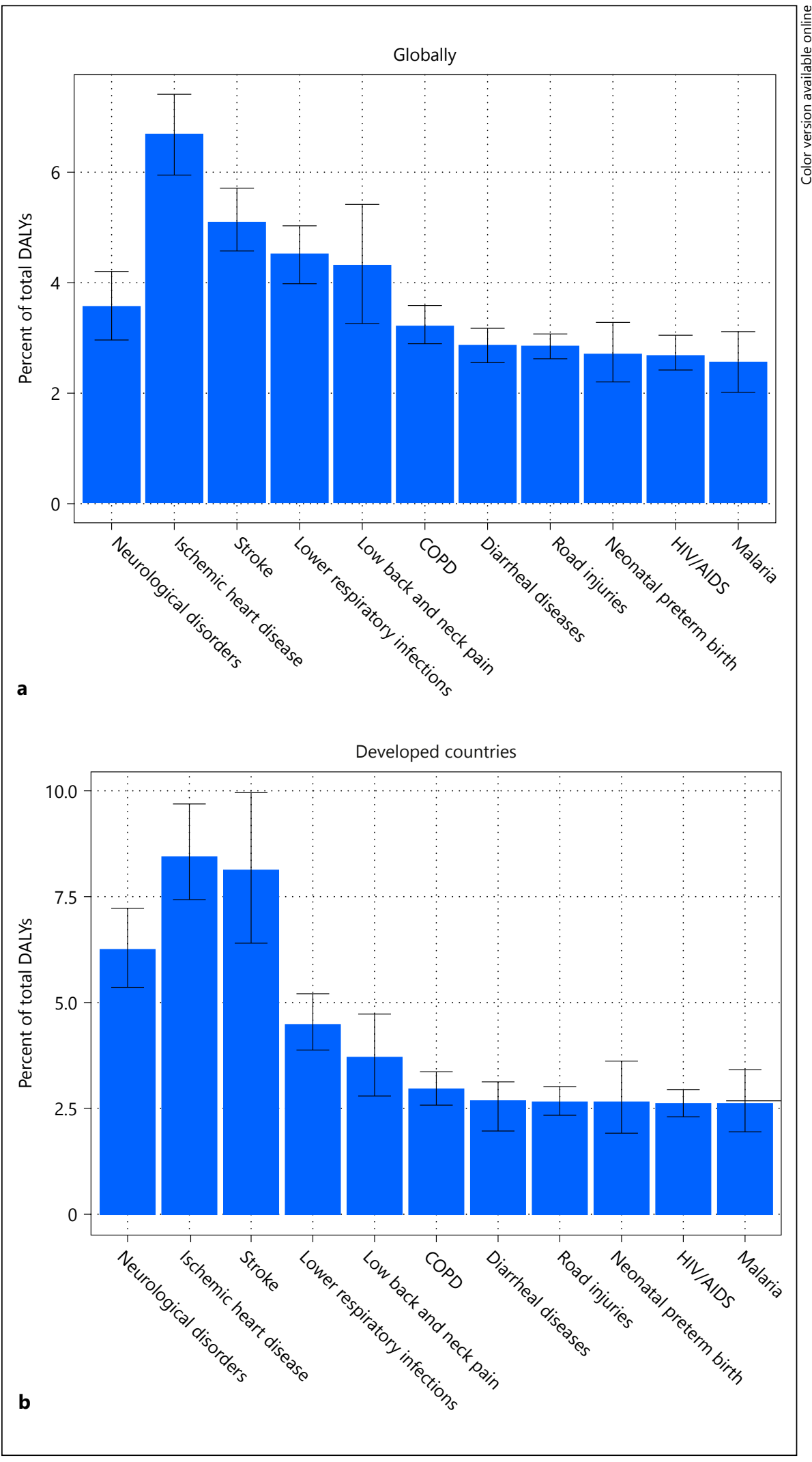

(For figure c see next page.) 


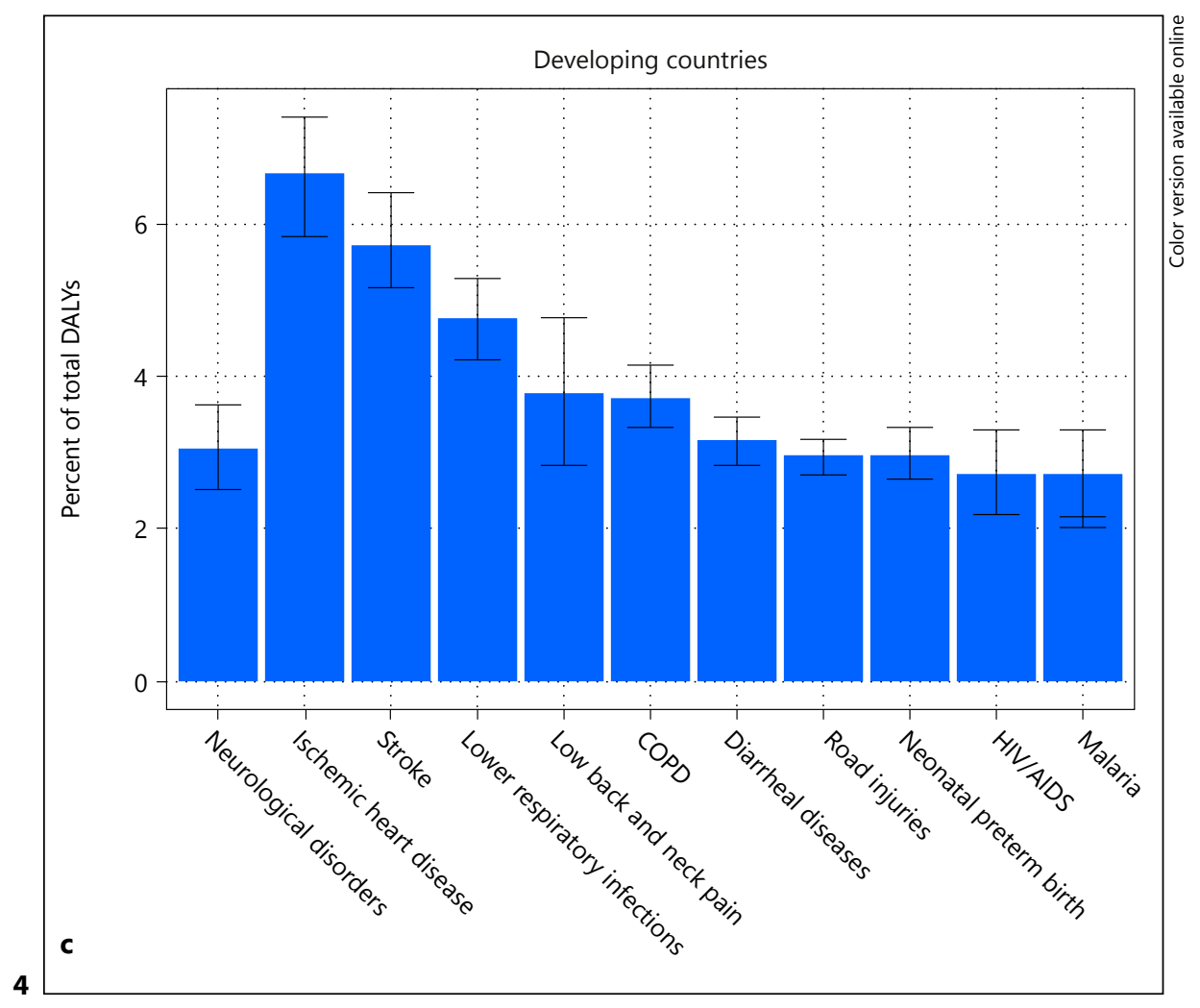

in developing countries. We demonstrated that globally, from 1990 to 2013, there was a statistically significant increase in HS prevalence rates and in developed countries the increase in the prevalence rates was statistically significant for both IS and HS, although the prevalence of IS decreased after 2005. While the increase in the prevalence of HS in developing countries may be related to the high rate of undetected and/or poorly controlled arterial hypertension [12-15], stroke incidence increase [11] or even better detection of stroke in those countries, the increase in the prevalence of IS and HS in developed countries could be related to the improvements in acute stroke care, or more effective secondary prevention and greater identification of minor stroke cases (including wider use of advanced neuroimaging) [16], which is highly dependent on universal access to primary care $[17,18]$.

We also found that proportional contribution of DALYs and deaths due to stroke to all diseases has increased from 1990 (3.54\% (95\% UI 3.11-4.00) and 9.66\% (95\% UI 8.47-10.70), respectively) to 2013 (4.62\% (95\% UI 4.01-5.30) and $11.75 \%$ (95\% UI 10.45-13.31), respectively) with a diverging trend in developed and developing countries: a statistically significant increase of the proportional contribution of stroke-related DALYs and stroke-related deaths in developing countries, and a trend, though not statistically significant, toward reduction of the proportional contribution of stroke-related DALYs and stroke-related deaths in developed countries. Apart from health transition in developing nations, this may also be explained by the widening gap in stroke prevention and management between developed and developing countries [19-21]. It may emphasize the pressing need for improving stroke prevention and treatment across the world [22]. The results of GBD 2013 showed the rise in the number of people with multi-morbidity and sequelae driven by longevity. Multi-morbidity has major implications for health systems and research, including stroke community $[23,24]$.

Although this research was based on the most comprehensive stroke epidemiological data relevant to the 19902013 study period and utilized the latest advances in the DisMod-MR stroke modeling techniques, it was not free from some limitations. Because death data were not available for all countries, mortality estimates relied on geospatial statistical models based on country-specific information. There was still lack of sufficient epidemiological data on stroke in most countries of the world, particularly in developing nations. To address this limitation, we used 


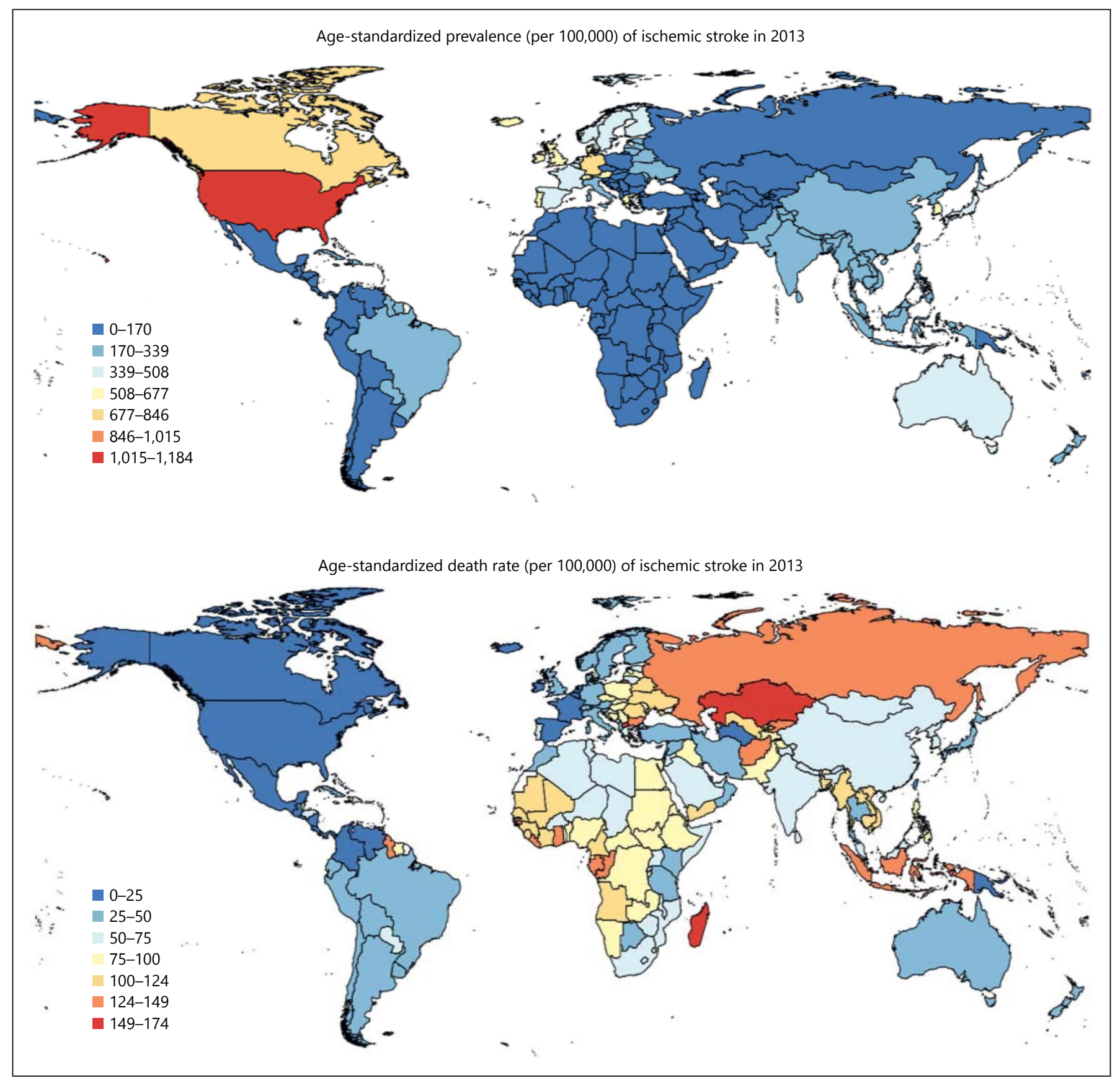

Fig. 5. Age-standardized prevalence and mortality of IS per 100,000 person-years in various regions in 2013.

country-level covariate data and applied out-of-sample validity testing to assess mortality model performance. Statistical models of stroke incidence and prevalence also relied on Bayesian methods and all available data on incidence and prevalence for each type of stroke. In particular, data from subnational regions were often assumed to represent an entire country. Although data were borrowed across time, which may blunt actual trends in disease epidemiology, the initial findings were reviewed by a global network of stroke experts to assess plausibility, and then subsequent refinements were made to data sources and modeling strategies based on their comments and suggestions. It should also be noted that current estimates combine intracerebral hemorrhage with other more rare stroke 


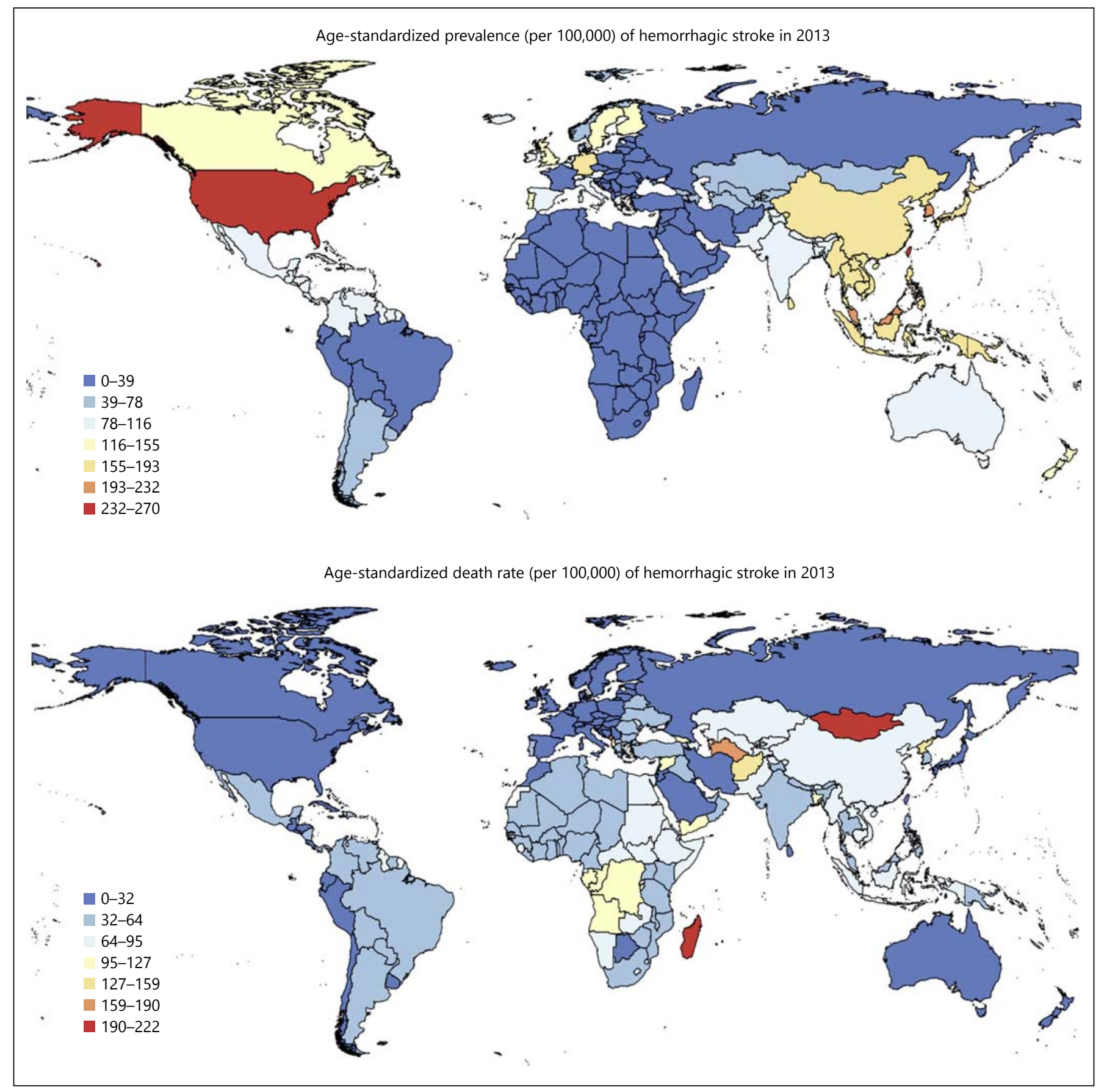

Fig. 6. Age-standardized prevalence and mortality of HS per 100,000 person-years in various regions in 2013.

types (such as subarachnoid hemorrhage) to better reflect the burden due to HS. To accurately reflect total disease burden, analysis was not limited to first-ever-in-a-lifetime strokes but also reflects all recurrent events. We were not able to report country-level incidence data because modeling at this level of detail is still under development. The
GBD 2015 study estimates will also include estimates of total stroke incidence (IS and HS combined) that we were not able to provide for this paper. Strengths of the study include the utilization of the latest advances in the DisMod stroke modeling techniques and a more comprehensive data set available for the analysis after 2010 . 
Stroke is currently the second largest contributor to DALYs after ischemic heart disease globally in developing countries, and it is third largest contributor to DALYs in developed countries (after ischemic heart disease and low back and neck pain). This emphasizes the importance of stroke as a leading global health problem that requires urgent and sustained attention from governments, healthcare policy makers, international agencies, clinicians, public health specialists and individual citizens. If the current trend in stroke burden continues, the UN Global target to reduce premature mortality due to non-communicable diseases [22] (including stroke) by $25 \%$ by 2025 will not be met. More efficient stroke prevention strategies and improved organization of stroke systems of care are urgently needed to halt and eventually reverse the stroke pandemic. Although there were improvements in the rates of DALYs, mortality and incidence rates from 1990 to 2013, the magnitude of the differences between these changes in developed and developing countries increased over time, suggesting disparities in the access to and quality of primary and secondary prevention and acute care between these countries. Therefore, there is an urgent need for action to negate these disparities.

\section{Contributions}

V.L.F., G.A.R. and C.M. developed the study concept and oversaw the research. V.L.F., R.K. and G.A.R. undertook reviews of studies. M.H.F., M.N. and P.P. provided statistical analysis of the data and produced graphs. V.L.F. wrote the first draft of the report. M.H.F., M.N. and C.M. developed the statistical model and wrote a section on statistical analysis. All authors contributed to the critical revision of the manuscript for important intellectual content.

\section{Disclosure Statement}

All the authors declare that they have no conflict of interest.

\section{Acknowledgments}

This work was undertaken as a part of the Global Burden of Diseases, Injuries, and Risk Factors (GBD 2013) study. The results in this paper are prepared independently of the final estimates of the GBD 2010 study. We thank Sarah Safranek, University of Washington Health Sciences Library, for her help in developing the systematic review literature search strategies; Michael F. MacIntyre, Brittany Wurtz, Caitlyn Steiner and Summer Ohno, University of Washington Institute for Health Metrics and Evaluation, for research coordination; Ryan M. Barber, University of Washington Institute for Health Metrics and Evaluation, for creating figures and tables for the paper; Hannah Gardener, University of Miami, for helping reviewing some studies; and Helen McDonald, AUT University National Institute for Stroke and Applied Neurosciences, for organising teleconferences and secretarial support. The authors alone are responsible for the views expressed in this publication.

\section{Role of the Funding Source}

This study was funded by the Bill and Melinda Gates Foundation. The sponsor of the study had no role in the study design, data collection, data analysis, data interpretation or writing of the report. The Writing and GBD 2013 Global Analysis Group had access to all data sources and has responsibility for the content of the report and the decision to submit for publication.

\section{Disclaimer}

The views expressed in this article are those of the authors and do not necessarily represent the views of the National Heart, Lung, and Blood Institute, National Institute of Mental Health, National Institutes of Health, or the U.S. Department of Health and Human Services.

\section{Appendix}

\section{GBD 2013 Writing Group (in Alphabetical Order)}

Foad Abd-Allah, Semaw Ferede Abera, Rufus Olusola Akinyemi, Rustam Al-Shahi Salman, Craig S. Anderson, Maria Cecilia Bahit, Amitava Banerjee, Sanjay Basu, Norman J. Beauchamp, Natan M. Bornstein, Michael Brainin, Norberto Luiz Cabral, Ismael Campos-Nonato, Valeria Caso, Ferrán Catalá-López, Rajiv Chowdhury, Hanne K. Christensen, Myles D. Connor, Gabrielle deVeber, Samath D. Dharmaratne, Klara Dokova, Geoffrey Donnan, Matthias Endres, Jefferson Gomes Fernandes, Fortuné Gankpé, Johanna M. Geleijnse, Richard F. Gillium, Maurice Giroud, Randah R. Hamadeh, Graeme J. Hankey, Panniyammakal Jeemon, Jost B. Jonas, Dhruv S. Kazi, Andre Pascal Kengne, Daniel Kim, Brett M. Kissela, Yoshihiro Kokubo, Soewarta Kosen, Michael Kravchenko, Pablo M. Lavados, Ming Liu, Paulo A. Lotufo, Mark T. Mackay, Reza Malekzadeh, Man Mohan Mehndiratta, Yohannes Adama Melaku, Awoke Misganaw, Devina Nand, Michael Piradov, Farshad Pourmalek, David Rojas-Rueda, Nobhojit Roy, Ramesh Sahathevan, Uchechukwu K.A. Sampson, Nikolay Shamalov, Kevin N. Sheth, Yukito Shinohara, Ivy Shiue, Michael Soljak, Luciano A. Sposato, Konstantinos Stroumpoulis, David Tanne, Amanda G. Thrift, David Lawrence Tirschwell, Yuri Varakin, Narayanaswamy Venketasubramanian, Vasiliy Victorovich Vlassov, Wenzhi Wang, Ronny Westerman, Charles Wolfe, Chuanhua Yu, Kim Yunjin, on behalf of the GBD 2013 Stroke Panel Experts Group.

\section{Author Affiliations}

Foad Abd-Allah, MD, Department of Neurology, Cairo University, Cairo, Egypt.

Semaw Ferede Abera, BSc-Public Health, MSc-Biostatistics and Health Informatics, Department of Epidemiology and Biostatistics, School of Public Health, College of Health Sciences, Mekelle University, Mek'ele, Ethiopia. 
Rufus Olusola Akinyemi, MBBS (Ib), MSc (Ib), PhD (Newcastle), MWACP, FMCP (Neurol), Institute for Advanced Medical Research and Training College of Medicine, University of Ibadan, Ibadan, Nigeria.

Rustam Al-Shahi Salman, MA, PhD, FRCP (Edin), Centre for Clinical Brain Sciences, University of Edinburgh, Edinburgh, Scotland.

Craig S. Anderson, MD, PhD, FRACP, The George Institute for Global Health, The University of Sydney, Royal Prince Alfred Hospital, Sydney, Australia.

Maria Cecilia Bahit, MD, Cardiology Department, INECO Neurociencias Rosario, Rosario, Sante Fe, Argentina.

Amitava Banerjee, Farr Institute of Health Informatics Research, University College London, London, UK.

Sanjay Basu, PhD, Stanford Prevention Research Center, Stanford University School of Medicine, Stanford, Calif., USA.

Norman J. Beauchamp, MD, MHS, Department of Radiology, University of Washington, Seattle, Wash., USA.

Natan Bornstein, MD, FAHA, FESO, Tel Aviv Sourasky Medical Center, Tel Aviv, Israel.

Michael Brainin, MD, PhD, FESO, FAHA, Department of Clinical Neurology, Danube University Krems and Karl Landsteiner University, Krems, Austria.

Norberto Luiz Cabral, MD, PhD, Joinville Stroke Registry, Clinica Neurológica de Joinville, University of Joinville Region, Brazil.

Ismael Campos-Nonato, Harvard School of Public Health, Nutrition Department, Boston, Mass., USA.

Valeria Caso, MD, PhD, Stroke Unit, University of Perugia, Perugia, Italy.

Ferrán Catalá-López, PhD, MPH, Department of Medicine, University of Valencia, CIBERSAM/INCLIVA, Valencia, Spain.

Rajiv Chowdhury, MD, PhD, Department of Public Health and Primary Care, School of Clinical Medicine, University of Cambridge, Cambridge, UK.

Hanne K. Christensen, MD, PhD, DMSc, University of Copenhagen and Bispebjerg Hospital, Copenhagen, Denmark.

Myles D. Connor, MD, Centre for Clinical Brain Sciences, The University of Edinburgh, Edinburgh, Scotland.

Gabrielle deVeber, MD, University of Toronto, Children's Stroke Program, Division of Neurology, and the Research Institute, Hospital for Sick Children, Toronto, Canada.

Samath D. Dharmaratne, MBBS, MSc, MD, Department of Community Medicine, Faculty of Medicine, University of Peradeniya, Peradeniya, Sri Lanka.

Klara Dokova, MD, PhD, Department of Social Medicine and Health Care Organization, Faculty of Public Health, Medical University of Varna, Varna, Bulgaria.

Geoffrey Donnan, MBBS, MD, FRACP, FRCP (Edin), The Florey Institute of Neuroscience and Mental Health and School of Neurology, University of Melbourne, Austin Hospital campus, Parkville, Vic., Australia.

Matthias Endres, MD, Klinik und Hochschulambulanz für Neurologie, Charité Universitätsmedizin Berlin, Berlin, Germany.

Jefferson Gomes Fernandes, MD, PhD, School of Health Education and Sciences, German Hospital Oswaldo Cruz, São Paulo, Brazil.

Fortuné Gankpé, MD, MSc, CHU Hassan II, Fez, Morocco.

Johanna M. Geleijnse, PhD, FAHA, MSc (Epidemiology), Division of Human Nutrition/Epidemiology, Wageningen University, Wageningen, The Netherlands.
Richard F. Gillum, MD, Department of Internal Medicine and Department of Community and Family Medicine, Howard University College of Medicine, Wash., USA.

Maurice Giroud, MD, Dijon Stroke Registry, Service de Neurologie, Dijon, France.

Randah R. Hamadeh, BSc, MSc, DPhil (Oxon), Department of Family and Community Medicine, College of Medicine and Medical Sciences, Arabian Gulf University, Manama, Bahrain.

Graeme J. Hankey, MD, FRACP, FRCP, FAHA, School of Medicine and Pharmacology, The University of Western Australia, Crawley, and Western Aiustralian Neuroscience Research Institute, Nedlands, WA, Australia.

Panniyammakal Jeemon, $\mathrm{PhD}$, Centre for Control of Chronic Conditions, Public Health Foundation of India and Centre for Chronic Disease Control, New Delhi, India.

Jost B. Jonas, MD, Department of Ophthalmology, Medical Faculty Mannheim of the University of Heidelberg, Heidelberg, Germany.

Dhruv S. Kazi, MD, MS, UCSF School of Medicine, University of California, San Francisco, Calif., USA.

Andre Pascal Kengne, MD, PhD, South African Medical Research Council, Parrow Valley, Cape Town, South Africa.

Daniel Kim, MD, DrPH, Department of Health Sciences, Northeastern University, Boston, USA.

Brett M. Kissela, MD, MS, Department of Neurology and Rehabilitation Medicine, University of Cincinnati, Ohio., USA.

Yoshihiro Kokubo, MD, PhD, FACC, FAHA, FESC, FESO, FJSH, Department of Preventive Cardiology, National Cerebral and Cardiovascular Center, Osaka, Japan.

Soewarta Kosen, MD, Health Economics and Policy Analysis Unit, National Institute of Health Research and Development (NIHRD), Jakarta, Indonesia.

Michael Kravchenko, MD, Scientific Neurological Center, Moscow, Russia.

Pablo M. Lavados, MD, MPH, Servicio de Neurología, Departamento de Medicina, Clínica Alemana de Santiago-Universidad del Desarrollo and Universidad de Chile, Santiago, Chile.

Ming Liu, MD, PhD, Professor of Neurology, Center of Cerebrovascular Diseases, Department of Neurology, West China Hospital, Sichuan University, Chengdu, China.

Paulo A. Lotufo, MD, DrPH, Universidade de São Paulo Medical School and Centre for Clinical and Epidemiological Research, University of Sao Paulo, Sao Paulo, Brazil.

Mark T. Mackay, MBBS, Department of Neurology, Royal Children's Hospital Melbourne and Murdoch Childrens Research Institute, Parkville, Australia.

Reza Malekzadeh, MD, Digestive Disease Research Institute, Tehran University of Medical Sciences, Shariati Hospital, Tehran, Iran.

Man Mohan Mehndiratta, MD, DNB, DM (Neurology) FAAN, FANA, FAMS, FRCP (London, Edinburgh, Glasgow) FICP, FIAN, FIACM, Department of Neurology, Janakpuri Super Speciality Hospital, New Delhi, India.

Yohannes Adama Melaku, MPH, School of Public Health, Mekelle University, Mekele, Ethiopia.

Awoke Misganaw, Institute for Health Metrics and Evaluation, University of Washington, Seattle, Wash., USA.

Devina Nand, MBBS, PGDPH, PGCTT, MPH (Deans Honours), Health Information Unit, Dinem House, Toorak, Suva, Republic of Fiji. 
Michael Piradov, MD, PhD, Scientific Neurological Center, Moscow, Russia.

Farshad Pourmalek, MD, MPH, PhD, School of Population and Public Health, University of British Columbia, Vancouver, Canada.

David Rojas-Rueda, MD, MPH, PhD, Centre for Research in Environmental Epidemiology, ISGlobal, Barcelona, Spain.

Nobhojit Roy, MS (Gen Surg), MPH, Environmental Health Resource Hub, School of Habitat Studies, Tata Institute of Social sciences, Mumbai, India.

Ramesh Sahathevan, MD, PhD, UKM Medical Centre, Kuala Lumpur, Malaysia; and Calvary Healthcare, Bruce, ACT, Australia.

Uchechukwu K.A. Sampson, MD, MSc, MBA, MPH, MS, FACC, Center for Translation Research and Implementation Science (CTRIS), National Heart, Lung, and Blood Institute, National Institutes of Health, Bethesda, Maryland, USA.

Nikolay Shamalov, MD, Meditsinskiy Tsentr Gbou VPO Rnimu im. N.I. Pirogova Minzdrava Rossii, Moscow, Russia.

Kevin N. Sheth, Division of Neurocritical Care and Emergency Department of Neurology Neurosciences Intensive Care Unit, Yale School of Medicine \& Yale New Haven Hospital, USA.

Yukito Shinohara, MD, PhD, FAHA, Tachikawa Hospital, Tokyo, Japan.

Ivy Shiue, PhD, Faculty of Health and Life Sciences, Northumbria University, Newcastle upon Tyne, UK.

Michael Soljak, MD, School of Public Health, Imperial College, London, England.

Luciano A. Sposato, MD, MBA, FAHA, Department of Clinical Neurological Sciences. London Health Sciences Centre, Western University, London, ON. Canada.

Konstantinos Stroumpoulis, MD, PhD, Anesthesiology Department, Alexandra General Hospital of Athens, Athens, Greece.

David Tanne, MD, Chaim Sheba Medical Center and Tel Aviv University, Tel Aviv, Israel.

Amanda G. Thrift, BSc (Hons), PhD, PGDipBiostat, Stroke and Ageing Research (STARC), Department of Medicine, School of Clinical Sciences at Monash Health, Monash University, Clayton, Vic., Australia.

David Lawrence Tirschwell, MD, MSc, FAHA, FAAN, University of Washington School of Medicine, UW Medicine Comprehensive Stroke Center, Harborview Medical Center, Seattle, Wash., USA.

Yuri Varakin, MD, PhD, Research Center of Neurology, Moscow, Russia.

Narayanaswamy Venketasubramanian, MBBS, MMed (Int Med), DLHTM (Epidemiology), FRCP (Edin), Raffles Neuroscience Centre, Raffles Hospital, Singapore, Singapore.

Vasiliy Victorovich Vlassov, MD, National Research University Higher School of Economics, Moscow, Russia.

Wenzhi Wang, MD, Beijing Neurosurgical Institute, Beijing, China.

Ronny Westerman, PhD, Competence Center Mortality-Follow-Up of the German National Cohort, Federal Institute for Population Research, Wiesbaden, Germany.

Charles Wolfe, MBBS, MRCOG, MD, MFPH, FFPH, FRCOG, Division of Health and Social Care Research, King's College London, London, UK; and National Institute for Health Research Comprehensive Biomedical Research Centre, Guy's \& St., Thomas' NHS Foundation Trust and King's College London, London, UK.

Chuanhua $\mathrm{Yu}, \mathrm{PhD}$, Prof. of Biostatistics, Wuhan University, Wuhan, PR China.
Kim Yunjin, MD, PhD, Faculty of Chinese Medicine Southern Malaysia, Southern University College, Johor, Malaysia.

\section{GBD 2013 Stroke Panel Experts Group (in Alphabetical Order} by Country)

Argentina (Maria Cecilia Bahit); Australia (Amanda G. Thrift, Atte Meretoja, Bill Stavreski, Craig S. Anderson, Edwin Pearse, Geoffrey Donnan, Graeme J. Hankey, Mark T. Mackay, Stephen Davis, Zanfina Ademi); Austria (Michael Brainin); Azerbaijan (Tural Guliyev); Bahrain (Randah R. Hamadeh); Barbados (Heather Harewood, Karen Springer); Brazil (Iuri da Costa Leite, Jefferson Gomes Fernandes, Norberto Luiz Cabral, Paulo A. Lotufo); Bulgaria (Klara Dokova); Canada (Farshad Pourmalek, Gabrielle deVeber, Luciano A. Sposato, M. Patrice Lindsay, Patricia M. Riccio); Chile (Pablo M. Lavados); China (Bin Li, Chuanhua Yu, Guohong Jiang, Jixiang Ma, Maigeng Zhou, Ming Liu, Shankuan Zhu, Wenzhi Wang, Xiaofeng Liang, Yong Zhang); Colombia (Gabriel Alcalá-Cerra); Denmark (Hanne K. Christensen, Thomas Truelsen); Egypt (Foad Abd-Allah); Ethiopia (Awoke Temesgen, Berhe Weldearegawi Sahle, Semaw Ferede Abera, Yohannes Adama Melaku); Fiji (Devina Nand); France (Maurice Giroud); Germany (Jost B. Jonas, Matthias Endres, Ronny Westerman); Greece (Konstantinos Stroumpoulis); India (Dorairaj Prabhakaran, Jeyaraj Durai Pandian, Man Mohan Mehndiratta, Nobhojit Roy, Panniyammakal Jeemon, Rajeev Gupta, Vasanthan Rajagopalan); Indonesia (Soewarta Kosen, Tati Suryati Warouw); Iran (Reza Malekzadeh); Ireland (Martin J. O'Donnell); Israel (David Tanne, Natan M. Bornstein); Italy (Stefano Ricci, Valeria Caso); Japan (Yoshihiro Kokubo, Yukito Shinohara); Jordan (Majed Masoud Asad); Kenya (Vitalis Kizito Bwire); Korea (Sun Ha Jee, Young-Ho Khang); Malaysia (Kim Yunjin, Ramesh Sahathevan); Mexico (Ismael Campos-Nonato); Morocco (Fortuné Gankpé); Myanmar (Chaw Yin Myint); Netherlands (Johanna M. Geleijnse); New Zealand (Priya Parmar, Rita Krishnamurthi, Suzanne Barker-Collo, Valery Feigin); Nigeria (Rufus Olusola Akinyemi); Norway (Ole Norheim); Qatar (Shams Eldin Khalifa); Russia (Michael Kravchenko, Michael Piradov, Nikolay Shalamov, Vasiliy Victorovich Vlassov, Yuri Varakin); Rwanda (Jean De Dieu Ngirabega, Jean Pierre Nyemazi, Marie Aimee Muhimpundu); Saudi Arabia (Mohammad Saeedi, Neeraj Bedi); Singapore (Narayanaswamy Venketasubramanian); South Africa (Andre Pascal Kengne); Spain (David Rojas-Rueda, Ferrán Catalá-López); Sri Lanka (Samath D. Dharmaratne); Sweden (Bo Norrving, Rasmus Havmoeller); Uganda (Leo Atwine); United Kingdom (Amitava Banerjee, Charles Wolfe, Derrick A. Bennett, Finbar O'Callaghan, Ivy Shiue, Julia A. Critchley, Majid Ezzati, Michael Soljak, Myles D. Connor, Peter M. Rothwell, Rajiv Chowdhury, Rustam Al-Shahi Salman, William Whiteley, Zhengming Chen); Uruguay (Mercedes Colomar); USA (Adnan M. Durrani, Anand Dayama, Andrew E. Moran, Awoke Misganaw, Brett M. Kissela, Catherine Amlie-Lefond, Catherine O. Johnson, Cheng Huang, Christopher J.L. Murray, Chugh Sumeet, Daniel Kim, David K. Cundiff, David Lawrence Tirschwell, Dhruv S. Kazi, Dima Qato, Edmond Kato Kabagambe, Eric Ding, Gene Bukhman, Gene Kwan, George A. Mensah, George Thurston, Grant Nguyen, Gregory A. Roth, Josef Coresh, Kate Lefondulq, Kevin N. Sheth, Matthew A. Corriere, Mohammad H. Forouzanfar, Mohsen Naghavi, Nana Mainoo, Norman J. Beauchamp, Ralph L. Sacco, Richard F. Gillum, Sanjay Basu, Stephen M. Schwartz, Sumeet Chugh, Teresa Fung, Theo Vos, Tim E. Byers, Uchechukwu K.A. Sampson, Walter A. Rocca, Warren Lo). 


\section{References}

$\triangleright_{1}$ Feigin VL, Forouzanfar MH, Krishnamurthi R, Mensah GA, Connor M, Bennett DA, Moran AE, Sacco RL, Anderson L, Truelsen T, O'Donnell M, Venketasubramanian N, Barker-Collo S, Lawes CM, Wang W, Shinohara Y, Witt E, Ezzati M, Naghavi M, Murray C: Global and regional burden of stroke during 1990-2010: findings from the Global Burden of Disease study 2010. Lancet 2014;383:245254.

-2 Krishnamurthi RV, Feigin VL, Forouzanfar $\mathrm{MH}$, Mensah GA, Connor M, Bennett DA, Moran AE, Sacco RL, Anderson LM, Truelsen $\mathrm{T}$, O'Donnell M, Venketasubramanian $\mathrm{N}$, Barker-Collo S, Lawes CM, Wang W, Shinohara Y, Witt E, Ezzati M, Naghavi M, Murray C: Global and regional burden of first-ever ischaemic and haemorrhagic stroke during 1990-2010: findings from the Global Burden of Disease study 2010. Lancet Glob Health 2013;1:e259-e281.

-3 Roth GA, Forouzanfar MH, Moran AE, Barber R, Nguyen G, Feigin VL, Naghavi M, Mensah GA, Murray CJ: Demographic and epidemiologic drivers of global cardiovascular mortality. N Engl J Med 2015;372:13331341.

4 An integrative metaregression framework for descriptive epidemiology. University of Washington Press, 2015.

5 Global Burden of Disease Study 2013 Collaborators: Global, regional, and national incidence, prevalence, and years lived with disability for 301 acute and chronic diseases and injuries in 188 countries, 1990-2013: a systematic analysis for the Global Burden of Disease study 2013. Lancet 2015;386:743800.

6 GBD 2013 Mortality and Causes of Death Collaborators: Global, regional, and national age-sex specific all-cause and cause-specific mortality for 240 causes of death, 1990-2013: a systematic analysis for the Global Burden of Disease study 2013. Lancet 2015;385:117171.
7 Roth GA, Johnson CO, Nguyen G, Naghavi $\mathrm{M}$, Feigin VL, Murray CJL, Forouzanfar MH, Vos T: Methods for estimating the global burden of cerebrovascular diseases. Neuroepidemiology 2015;45:146-151.

8 Aho K, Harmsen P, Hatano S, Marquardsen J, Smirnov VE, Strasser T: Cerebrovascular disease in the community: results of a WHO collaborative study. Bull World Health Organ 1980;58:113-130.

9 Flaxman AD, Vos T, Murray CJ (eds): An Integrative Metaregression Framework for Descriptive Epidemiology, University of Washington Press, 2015. http://www.washington. edu/uwpress/search/books/FLAINT.html.

10 Global, regional, and national incidence, prevalence, and years lived with disability for 301 acute and chronic diseases and injuries in 188 countries, 1990-2013: a systematic analysis for the Global Burden of Disease study 2013. Lancet 2015;386:743-800.

-11 Feigin VL, Lawes CM, Bennett DA, BarkerCollo SL, Parag V: Worldwide stroke incidence and early case fatality reported in 56 population-based studies: a systematic review. Lancet Neurol 2009;8:355-369.

12 Maher D, Waswa L, Baisley K, Karabarinde A, Unwin N: Epidemiology of hypertension in low-income countries: a cross-sectional population-based survey in rural Uganda. J Hypertens 2011;29:1061-1068.

13 Ibrahim MM, Damasceno A: Hypertension in developing countries. Lancet 2012;380:611619.

14 Kumara WA, Perera T, Dissanayake M, Ranasinghe $\mathrm{P}$, Constantine GR: Prevalence and risk factors for resistant hypertension among hypertensive patients from a developing country. BMC Res Notes 2013;6:373.

15 Mehndiratta MM, Khan M, Mehndiratta P, Wasay M: Stroke in Asia: geographical variations and temporal trends. J Neurol Neurosurg Psychiatry 2014;85:1308-1312.
16 Bernick C, Kuller L, Dulberg C, Longstreth WT Jr, Manolio T, Beauchamp N, Price T: Silent MRI infarcts and the risk of future stroke: the cardiovascular health study. Neurology 2001;57:1222-1229.

17 Lee S, Shafe AC, Cowie MR: UK stroke incidence, mortality and cardiovascular risk management 1999-2008: time-trend analysis from the general practice research database. BMJ Open 2011;1:e000269.

18 Soljak M, Calderon-Larrañaga A, Sharma P, Cecil E, Bell D, Abi-Aad G, Majeed A: Does higher quality primary health care reduce stroke admissions? A national cross-sectional study. Br J Gen Pract 2011;61:e801-e807.

19 Xu G, Yin Q, Liu X: Narrowing the gap of stroke management between developed and developing countries: report of the fifth international stroke summit, Nanjing, China, July 17-19, 2009. Cerebrovasc Dis 2010;29:101-102.

20 Strong K, Mathers C, Bonita R: Preventing stroke: saving lives around the world. Lancet Neurol 2007;6:182-187.

21 Abd-Allah F, Moustafa RR: Burden of stroke in Egypt: current status and opportunities. Int J Stroke 2014;9:1105-1108.

22 United Nations General Assembly: Resolution adopted by the general assembly: $66 / 2$ : political declaration of the high-level meeting of the general assembly on the prevention and control of non-communicable diseases. http://www.who.int/nmh/events/un_ncd summit2011/political_declaration_en.pdf (adopted September 19, 2011).

23 Smith SM, Soubhi H, Fortin M, Hudon C, O'Dowd T: Managing patients with multimorbidity: systematic review of interventions in primary care and community settings. BMJ 2012;345:e5205.

24 Gallacher KI, Batty GD, McLean G, Mercer SW, Guthrie B, May CR, Langhorne P, Mair FS: Stroke, multimorbidity and polypharmacy in a nationally representative sample of $1,424,378$ patients in Scotland: implications for treatment burden. BMC Med 2014;12:151. 


\section{Erratum}

This erratum refers to the article by Feigin et al., entitled "Update on the global burden of ischemic and hemorrhagic stroke in 1990-2013: The GBD 2013 Study" [Neuroepidemiology 2015;45:161-176, DOI: 10.1159/000441085]. The authors regret that there are 3 errors in this paper.

In table 1, 1990 HS 3rd row, “27.9 (25.1-33.2), etc.” should be ignored.

On page 166, the 5th line from the bottom should read "and the second largest contributor to DALYs in developed countries (after ischemic heart disease), with ..."

On page 173, the first sentence at the top should read "Stroke is currently the second largest contributor to DALY after ischemic heart disease globally and in both developed and developing countries." 